Check for updates

Cite this: RSC Adv., 2019, 9, 2836

Received 21st November 2018

Accepted 23rd December 2018

DOI: $10.1039 / c 8 r a 09596 e$

rsc.li/rsc-advances

\section{Novel tunable super-tough materials from biodegradable polymer blends: nano-structuring through reactive extrusion $\dagger$}

\author{
Feng Wu, ${ }^{\text {ab }}$ Manjusri Misra (iD ${ }^{\text {ab }}$ and Amar K. Mohanty (D) *ab
}

Structuring blends on sub-micrometer scales, especially nano-scales, has a higher potential for improving their thermomechanical properties. Here, we propose a design strategy to fabricate compatible nanoblends by manipulating the reactions between two biodegradable polymers, e.g. polybutylene succinate (PBS) and polybutylene adipate terephthalate (PBAT), with extremely low free radical contents through reactive extrusion processing. Observed by transmission electron microscopy (TEM) and atomic force microscopy (AFM), it is found that PBAT is tightly surrounded by large amounts of PBS-PBAT co-polymers and dispersed in a PBS matrix with a particle size of less than $100 \mathrm{~nm}$. We show how impact strength and polymer moduli can be improved simultaneously by decreasing the small amount of dispersed phase into nano-scale (droplet or lamina structures). With 5 wt\% PBAT content in the PBS-PBAT blend, the notched impact strength of PBS is increased $1200 \%$ and the Young's modulus is increased $15 \%$. Through in situ rheological monitoring and Fourier-transform infrared spectroscopy (FTIR) studies, the reason why nanoblends can be formed in such a low amount of peroxide is illustrated. Our investigation most significantly indicates the transformation of the partially compatible PBS-PBAT micro-blend into a fully compatible PBS-PBAT through nano-structuring. This work addresses the importance of reaction rate and mechanism in favoring the formation of co-polymers rather than homo-polymer crosslinking or self-decomposition in polymer blend modification via reactive extrusion design.

\section{Introduction}

The development of super-toughened polymers with high impact strength possess great scientific significance and huge market potentials. This is because of the fact that high toughness can broaden the application of materials dramatically. ${ }^{1}$ One of the widely used polymers in packaging applications is polystyrene (PS), which was discovered in 1839 and the PS market attained great commercial success with the discovery of high impact polystyrene (HIPS). ${ }^{2,3}$ From "living polymerization" to "accepted commercialization", the HIPS family inspired scientists to develop high impact plastics via structure control. Extensive studies on the morphology of HIPS revealed the nanophase separation of these polymers, between the polystyrene (PS) and its co-polymer with polyisoprene (PI) or polybutadiene (PB). ${ }^{4}$ The nano-structuring is the key factor to obtain high impact strength. Therefore, the structuring of polymer blends

\footnotetext{
${ }^{a}$ Bioproduct Discovery and Development Centre, Department of Plant Agriculture, University of Guelph, Crop Science Building, Guelph, ON N1G 2W1, Ontario, Canada.E-mail: mohanty@uoguelph.ca

${ }^{b}$ School of Engineering, University of Guelph, Thornbrough Building, Guelph, ON N1G 2W1, Ontario, Canada

$\uparrow$ Electronic supplementary information (ESI) available. See DOI: 10.1039/c8ra09596e
}

or novel co-polymers on nano-scale by self-assembly of industrially available polymers raises much hope to fabricate the super-toughened materials.

At present, nearly $99 \%$ of all used plastics are nonbiodegradable and of which $79 \%$ are discarded and accumulated in landfills or in the natural environment as of $2015 .^{4}$ Roughly $12000 \mathrm{Mt}$ of plastic will be generated by 2050 if current production and management trends continue. ${ }^{5}$ This will become an increasingly pressing problem for governments, scientists and the general population. Therefore, the development of biodegradable polymers from renewable resources has increased worldwide. ${ }^{6}$ These biodegradable polymers are expected to replace traditional petroleum-based plastics in commodity and engineering applications. ${ }^{7,8}$ However, the amount of bio-plastic used in the world is significantly less than the potential market, due to the limited performance of important properties and higher cost of the few commodity biodegradable polymers in comparison to petrochemical plastics. To increase the use of biopolymers there is a need to improve their performance characteristics or to develop novel properties customized to satisfy specific applications. Taking polybutylene succinate (PBS) as an example, this material possesses high elongation, good biodegradable and high heat deflection temperature (HDT), ${ }^{9}$ however, the notched impact strength of the PBS is very low as reported $\left(40 \mathrm{~J} \mathrm{~m}^{-1}\right) .{ }^{10}$ If high 
impact strength PBS is developed, the materials can find widespread applications in markets.

Blending has long been considered to be a simpler and lower cost strategy to modify in bringing new properties to the polymer families. However, blending different polymers naturally tends to lead to phase separation on the macroscopic scale; the dispersed phase normally exists at the micrometer scale, and therefore it cannot maximize the properties of the different components. Consequently, substantial improvement of the thermal and mechanical properties such as high toughness, high stiffness and high-temperature creep resistance can only be achieved by improving the miscibility and dispersion state, especially at the nano-scale ${ }^{\mathbf{1 1}}$ - as confirmed by the HIPS story. According to the drop breakup theory in polymer blends, the diameter of the droplet of the dispersed phase $D$ can be determined by eqn (1): ${ }^{12}$

$$
D=\frac{4 \mathscr{T} \eta_{\mathrm{r}}{ }^{ \pm 0.84}}{\dot{\gamma} \eta_{\mathrm{m}}}
$$

where $\mathscr{T}$ is the interfacial tension, $\dot{\gamma}$ is the shear rate, $\eta_{\mathrm{m}}$ is the viscosity of main matrix, $\eta_{\mathrm{r}}=\frac{\eta_{\mathrm{d}}}{\eta_{\mathrm{m}}}, \eta_{\mathrm{d}}$ is the viscosity of the dispersed phase. Therefore, to decrease the diameter of the dispersed phase, the interfacial tension should be decreased, or increase the viscosity of the main matrix.

So far in polymer science, three main methods have been proposed to obtain nano-structure in polymer blends based on the above theory: (i) high-shear flow processing, ${ }^{13,14}$ (ii) ex situ A-B block polymer synthesized and blended with $\mathrm{A}$ and $\mathrm{B}$ homopolymers, ${ }^{\mathbf{1 5}}$ and (iii) polymerizing a monomer of one polymer in another matrix of macromolecules by reactive blending. ${ }^{16-19}$ Among them, reactive extrusion has been extensively researched for its simple and convenience in industrial processing. ${ }^{20}$ However, compared to the synthesis of large macromolecular structure from monomer via in situ reactive extrusion, fewer research has been performed on melt blending of existing high molecular weight polymers though reactive extrusion as a method to obtain nano-scale blends, especially in the biodegradable plastic fields. Also, how to achieve large scale manufacturing of nano-dispersion via reactive extrusion in controlling the polymer properties through peroxide usage is unclear in the scientific literature to the best of our knowledge. Pernot et al.' $\mathrm{s}^{\mathbf{2 1}}$ former research presents a principal design describing the formation of co-continuous nanostructure via lab-made polyethylene (PE) and polyamide (PA) in reactive blending. The research determined several fundamental facts as regards to structuring of nano-blends. For example, random graft co-polymer chains should be synthesized in situ by reactive blending two polymers which carry complementary reactive groups, followed by a coupling reaction that should be processed quickly.

Based on this fundamental work and previous research on nano-blends, here we present a general strategy to obtain high performance biodegradable blends (in the nano-scale) by improving miscibility for a pair of industrial biodegradable polymer pairs: PBS and PBAT. The chosen materials show different reactivity with peroxide at high temperatures, either crosslinking - the main mechanism - or decomposition. It is determined that biodegradable binary nano-blends exhibiting exceptional properties like super toughness with high modulus, high melt strength and improved thermal stability which are unattainable by using classical micro-blends. For example, our group's former research shown that microblending of $70 \mathrm{wt} \% \mathrm{PBS} / 30 \mathrm{wt} \%$ PBAT could improve the toughness of the PBS but meanwhile the modulus decreased a lot. ${ }^{22,23}$ Here we find that only $5 \mathrm{wt} \%$ PBAT can improve the toughness of PBS dramatically without the loss of high modulus of PBS when dispersing the PBAT into nano-scale. Several goals are achieved in our current research: (i) by carefully choosing the polymer pairs, nano-blends can be obtained by reactive extrusion and the mechanism of the nanoblends formation has been discussed here to guide the future nano material designing; (ii) the effects of the peroxide usage and its reaction with the polymer components is discussed; (iii) high impact strength and high melt strength PBS nano-blends can be processed like traditional blending without the need to change the current equipment or production speed; (iv) the low contents of peroxide initiator in this research meets the development of industrial environmentally-friendly processes; and furthermore biodegradable blends are expected to be developed base on the formulation and concept proposed here. ${ }^{24}$

\section{Experiment and methods}

\section{Materials}

Commercial PBS (Tunhe Th803s from Xinjiang Blueridge Tunhe Chemical Industry Co., Ltd. with weight-average molecular weight $\left(M_{\mathrm{w}}\right) \approx 2.3 \times 10^{4} \mathrm{~g} \mathrm{~mol}^{-1}$ ), PBAT (Tunhe Th801t from Xinjiang Blueridge Tunhe Chemical Industry Co., Ltd with $\left.M_{\mathrm{w}} \approx 7.50 \times 10^{4} \mathrm{~g} \mathrm{~mol}^{-1}\right)$, and peroxide(2,5-bis(tertbutylperoxy)-2,5-dimethylhexane, also known as 2,5-bis(tertbutylperoxy)-2,5-dimethylhexane or Luperox 101 from SigmaAldrich company) are used here. Chloroform $\left(\mathrm{CHCl}_{3}\right)$ for gel contents calculation is obtained from Sigma-Aldrich company and used without further purification. The PBS and PBAT are dried at $60{ }^{\circ} \mathrm{C}$ for $24 \mathrm{~h}$ before processing.

\section{Sample preparation}

The blending of PBS/PBAT in the presence of peroxide initiator is in situ extruded in a twin-screw extrusion (Leistritz Micro-27, Germany) with a screw diameter of $27 \mathrm{~mm}$ and an $L / D$ ratio of 48 and defined as nano-blends base on the morphological structure. The blends are subjected to extruder processing at temperatures of $175-190{ }^{\circ} \mathrm{C}$ (feeding to melting zone), with feeding speed at $5 \mathrm{~kg} \mathrm{~h}^{-1}$ and screw speed at $100 \mathrm{rpm}$. Following the extrusion process, the over-night dried pellets are injected into mechanical testing samples by a DSM explore system (DSM 15cc Twin Screw Micro-Compounder, Netherlands) for characterization. The injection is completed in $2 \mathrm{~min}$ at $175{ }^{\circ} \mathrm{C}$ with a screw speed of $100 \mathrm{rpm}$. The blends of PBS/PBAT without peroxide are defined as micro-blends and experience the same processing as above. 


\section{Microstructural observations}

The microstructure is characterized by scanning electron microscope (SEM), transmission electron microscopy (TEM) and atomic force microscopy (AFM). SEM is performed under a Phenom ProX (Netherlands) set to an accelerating voltage of $15 \mathrm{kV}$, and TEM is examined by using a JEOL JEM-1200 Ex II operating at an accelerating voltage of $80 \mathrm{kV}$. Ultra-thin specimens $(70 \mathrm{~nm})$ for TEM observation are cut from the middle section of molded samples in a perpendicular direction to the flow. The cut is performed under cryogenic conditions with a RMC PowerTome XL ultra-microtome with RMC CRX cryosectioning attachment using a diamond knife at $-80{ }^{\circ} \mathrm{C}$, and the film is retrieved on 300 mesh $\mathrm{Cu}$ grids. The specimens for AFM imaging are prepared by Leica Microtome (Germany) at room temperature and performed on a AFM instrument with Multimode 8 from Bruker Nano Inc., CA, U.S.A. The scanning is done at peak force tapping model at constant scanning rate of $0.401 \mathrm{~Hz}$.

\section{Rheological properties characterization}

The biopolymer (PBS and PBAT)/peroxide in situ reaction is performed on rheometer (Anton Paar MCR320) at different temperatures and different peroxide concentrations. The time sweep of the PBS/peroxide and PBAT/peroxide is performed at $175{ }^{\circ} \mathrm{C}$ with a fixed frequency of $1 \mathrm{~Hz}$. All the rheological testing is under $\mathrm{N}_{2}$ protection.

The evolution of the dynamic modulus and viscosity of the injection samples (micro-blends and nano-blends) are measured from 0.1 to $100 \mathrm{rad} \mathrm{s}^{-1}(1 \%$ strain) at different temperature $-180,190,200$ and $210{ }^{\circ} \mathrm{C}$. To calculate the flow activation energy of the blends, the zero-shear viscosity $\left(\eta_{0}\right)$ of the blends is obtained from the dynamic frequency sweep along with fits to the following Carreau model: ${ }^{25}$

$$
\eta(\dot{\gamma})=\frac{\eta_{0}}{\left[1+(\tau \dot{\gamma})^{2}\right]^{P}}
$$

where $\eta(\dot{\gamma})$ is the viscosity at shear rate $\dot{\gamma}, \tau$ is the relaxation time of the material, $P$ is determined by least-squares regression. The flow activation energy of the blends is obtained by linear fitting the zero shear viscosity as a function of the characterization temperatures.

\section{Differential scanning calorimetry (DSC)}

The crystallization and melting points of the materials are characterized by DSC (DSC Q200, TA, US) with a cooling and heating rate of $10{ }^{\circ} \mathrm{C} \mathrm{min}{ }^{-1}$ under $\mathrm{N}_{2}$ protection. The crystallinity of PBS is calculated by the following eqn (3):

$$
X_{\mathrm{c}}=\frac{\Delta H_{\mathrm{m}}}{w_{\mathrm{f}} \Delta H_{\mathrm{m}}^{\mathrm{o}}} \times 100 \%
$$

where the $\Delta H_{\mathrm{m}}$ is the melting enthalpies of PBS in the blends, $\Delta H_{\mathrm{m}}^{\mathrm{o}}$ is the melting enthalpies of assuming $100 \%$ crystalline PBS $\left(220 \mathrm{~J} \mathrm{~g}^{-1} \text { here }\right)^{26}$ and $w_{\mathrm{f}}$ is the weight fraction of PBS in the blend.

\section{FTIR spectrum characterization}

The biopolymer/peroxide in situ reacted samples (on rheometer) are characterized immediately in Fourier Transform Infrared (FTIR) spectrometer equipped with a smart orbit ATR (Nicolet 6700, Thermo Scientific).

\section{Mechanical and thermal mechanical testing}

Uniaxial tensile tests are performed using dog-bone shaped tensile samples with a gauge length of $25 \mathrm{~mm}$ and a width of

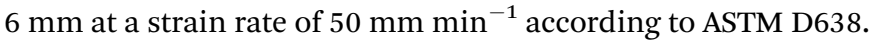
An extensometer is used to calibrate strain during uniform elongation. Notched impact tests are performed by a Testing Machine Inc. (TMI) instrument (Testing Machine Inc. US) according to ASTM D256. Each test is repeated at least 5 times.

Dynamic Mechanical Analysis (DMA) is carried out with a DMA Q800 from TA Instruments. A dual cantilever clamp is used at the frequency of $1 \mathrm{~Hz}$ and oscillating amplitude of 15 $\mathrm{mm}$. The samples are heated from -70 to $185{ }^{\circ} \mathrm{C}$ at a heating rate of $3{ }^{\circ} \mathrm{C} \mathrm{min}^{-1}$.

\section{Gel contents calculation}

The gel contents of the PBS/PBAT blends with peroxide are calculated by dissolve-extraction method in accordance with ASTM D2765. The blends are dissolved in chloroform for $48 \mathrm{~h}$ and the insoluble parts are extracted and dried for 3 days in a vacuum oven at $50{ }^{\circ} \mathrm{C}$ to remove the $\mathrm{CHCl}_{3}$. The residue of insoluble polymer is reported as \% gel content.

\section{Results and discussion}

\section{Super-toughened binary nano-blends}

The TEM images of the as-formed nano-blends with different PBAT contents are shown in Fig. 1. A clear, homogeneous dispersed phase made of nano-scale PBAT is observed with characteristic particle diameters below $100 \mathrm{~nm}$. The nanostructures are tailored by changing the composition ratio of the main PBS and dispersed PBAT. At low PBAT loadings (5 wt $\%$ PBAT shown in Fig. 1a and $10 \mathrm{wt} \%$ PBAT shown in Fig. 1b), the homogeneous phase is dispersed with a diameter of approximately $20 \mathrm{~nm}$. The dispersed nano-droplet develops to the nano-lamina structure with characteristics nano-dots of about $10 \mathrm{~nm}$ at $15 \mathrm{wt} \%$ PBAT loading as shown in Fig. 1c, and further develops to nano-droplet of $\sim 120 \mathrm{~nm}$ at $20 \mathrm{wt} \%$ PBAT as shown in Fig. 1d. Nano-droplet or nano-lamina structures can be obtained by changing the contents of the dispersed phase. It is hypothesized that PBS-PBAT co-polymer contents formed during reactive extrusion increased with increasing PBAT content. And the existence of the co-polymer decreases the interfacial tension between PBS and PBAT, thus decreases the diameter of the PBAT droplet based on eqn (1). However, the diameter of the PBAT droplet further increased to $\sim 120 \mathrm{~nm}$ resulting from the highest PBAT loadings (20 wt\%) as the diameter of the droplet increased with increasing disperse contents. $^{27}$

The blends are dissolved in chloroform to separate the possible homo-polymer gelation formed by the reactive 

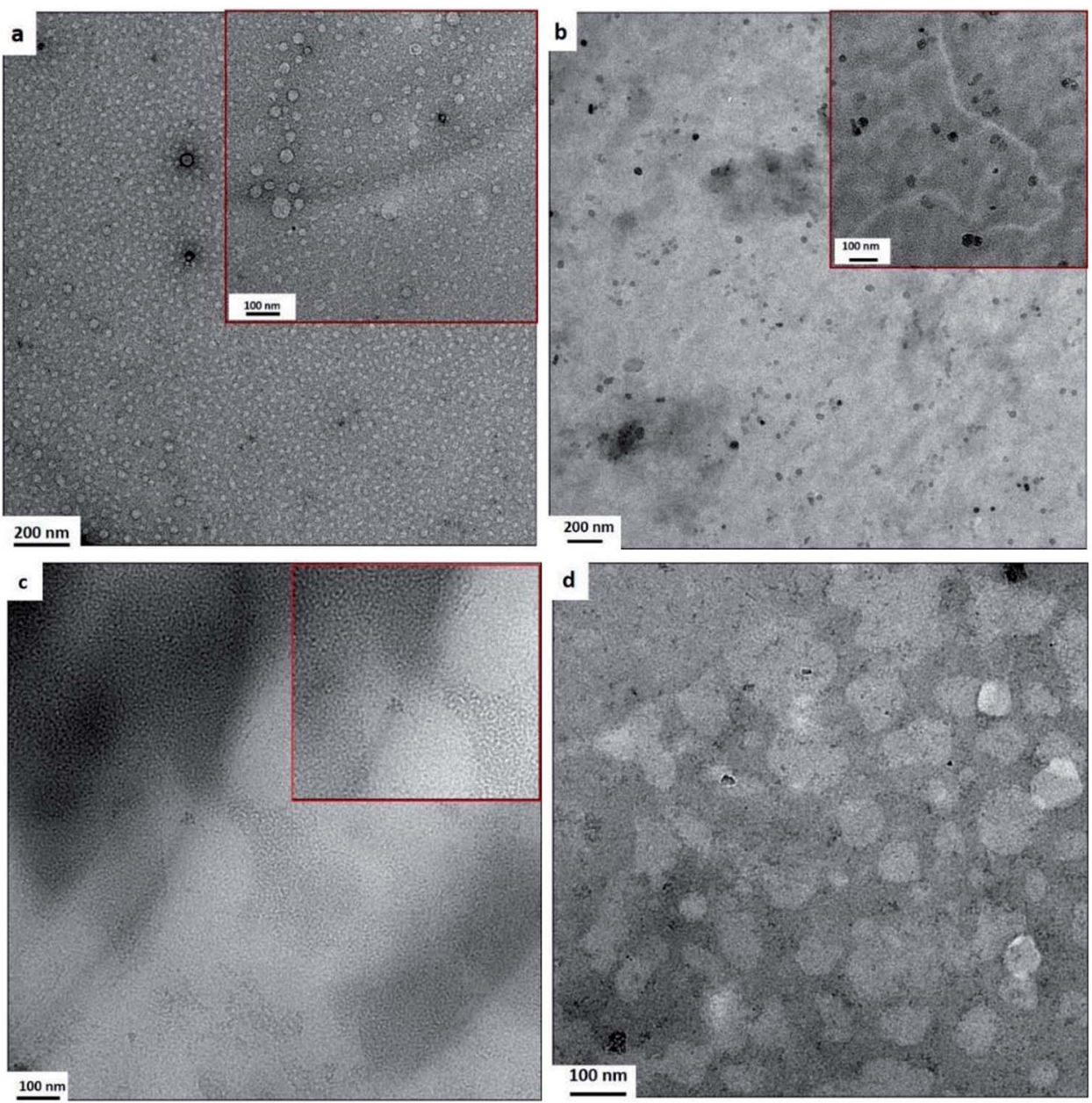

Fig. 1 Super-toughened nano-structured PBS/PBAT binary blend. Transmission electron micrographs for: (a) extruded 95PBS/5PBAT blends with 0.02 phr peroxide initiator; (b) extruded 90PBS/10PBAT blends with 0.02 phr peroxide initiator; (c) extruded 85PBS/15PBAT blends with 0.02 phr peroxide initiator; (d) extruded 80PBS/20PBAT blends with 0.02 phr peroxide initiator.

extrusion process. It is found that the gel contents (shown in Table S1†) decrease from $15.8 \%$ (5 wt\% PBAT) to $0 \%$ with increasing PBAT contents (15 wt $\%$ PBAT). The high gel contents of PBS95-PBAT5 blends with $0.02 \mathrm{phr}$ peroxide is mainly caused by the self-crosslinking of the PBS. With increasing PBAT contents, the homo-polymers' gelation is suppressed and PBSPBAT co-polymers are largely formed, resulting to the zero-gel at $15 \mathrm{wt} \%$ and higher PBAT. The low gel contents and strong shear thinning produced by the dynamic crosslinking network ensured the success of the melt processability of these materials in twin-screw extrusion, and the flow activation energy did increase slightly to $38.24 \mathrm{~kJ} \mathrm{~mol}^{-1}$ compared to the microblends of $28.68 \mathrm{~kJ} \mathrm{~mol}^{-1}$ (confirmed by zero-shear viscosity at different temperatures as shown in Fig. S1 $\dagger$ ), indicating the low energy processing of these nano-blends as the traditional processing of the micro-blends. This is because of the low dosage of peroxide used in the current research.

The influence of the morphology and PBAT contents on the mechanical performance of the blends is shown in Fig. 2. The notched impact strength of the blends to reflect the toughness is shown in Fig. 2a. The formation of the nano-structure results in a high impact strength up to $720 \mathrm{~J} \mathrm{~m}^{-1}$, which infers that the material displays super-toughness. ${ }^{28}$ The presence of only $5 \mathrm{wt} \%$ PBAT endows the nano-blends with much higher impact strength $\left(\sim 503 \mathrm{~J} \mathrm{~m}^{-1}\right)$ compared to that of $20 \mathrm{wt} \%$ PBAT in the micro-blends $\left(200 \mathrm{~J} \mathrm{~m}^{-1}\right)$, with a transition from complete break to hinge impact behaviour. The reduced use of PBAT ensure the super-toughened blends without sacrifice of the modulus of the PBS matrix, broadening the application of this blend.

In addition to the super-toughness qualities, the nano-blends also exhibit superior performance in other mechanical properties. The nano-blends exhibit higher tensile modulus compared to the micro-blends, as shown in Fig. 2b. Compared to the microblends where the tensile modulus is located between the pure PBS (613 $\pm 23 \mathrm{MPa})$ and PBAT (70 $\pm 7.5 \mathrm{MPa})$ which is demonstrated in Table S2 in ESI Section, $\uparrow$ the modulus of PBS95/PBAT5 nano-blends is higher than that of pure PBS, reaching $705 \pm$ $41 \mathrm{MPa}$, overcoming PBAT's shortcoming of low modulus. Furthermore, the moduli of nano-blends are higher than that of the micro-blends at same composition ratios. The unusually high modulus is believed to be a result of the reinforcement of the nanodispersion PBAT especially as the crystallinity of PBS decreased compared to the micro-blends because of the branching/ crosslinking (the crystallinity of the blends obtained from 1st 
(a)

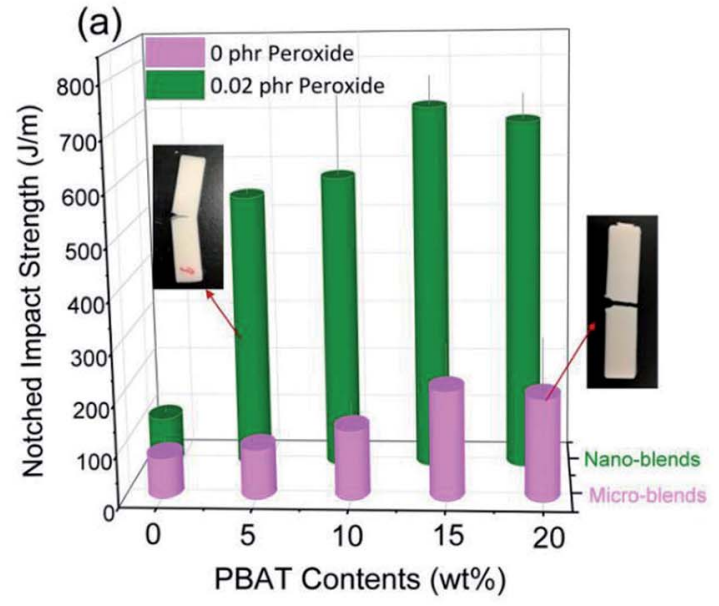

(b)

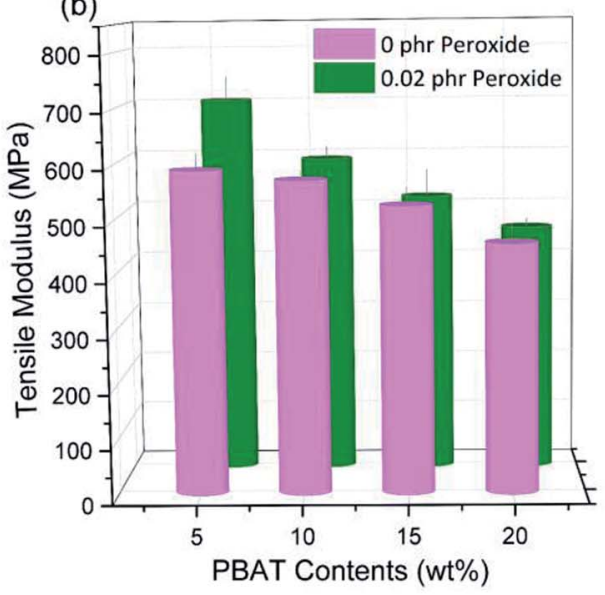

(d)

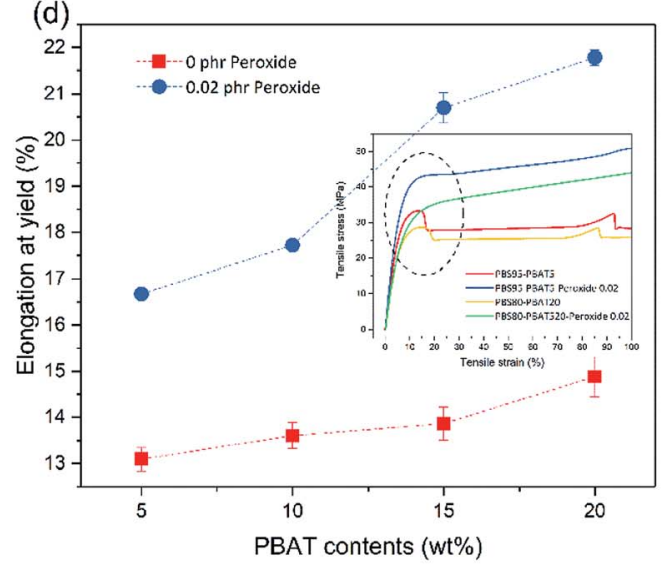

Fig. 2 Superior properties of the binary nano-blends. (a) Notched impact strength of the nano-blends and micro-blends with different PBAT contents, (b) dependence of tensile modulus on PBAT for micro and nano-blends; (c) dependence of elongation at break on PBAT contents for micro and nano-blends; (d) dependence of elongation at yield on PBAT contents for micro and nano-blends.

heating DSC curves are shown in Fig. S2 $\dagger$ ). The elongation at break $\left(\varepsilon_{\mathrm{b}}\right)$ of the blends is shown in Fig. $2 \mathrm{c}$ and it's found that that the $\varepsilon_{\mathrm{b}}$ of nano-blends decrease compared to the micro-blends, a characteristic of highly entangled materials. ${ }^{21}$ However, the elongation at yield of the nano-blends is much higher compared to the microblends, with a transition from plastic-like to hard elastomer-like fracture behaviour as illustrated in Fig. $2 \mathrm{~d}$.
Biodegradable materials with high melt strength are obtained in our research because of the high entanglement of the molecular chains by reactive branching, as indicated from the melt dynamic moduli in Fig. 3. The storage modulus $\left(\mathrm{G}^{\prime}\right)$ and loss modulus $\left(\mathrm{G}^{\prime \prime}\right)$ of the blends with and without peroxide are shown in Fig. 3a. The micro-blends of PBS/PBAT exhibits liquidlike viscoelastic behaviour with $G^{\prime \prime}$ higher than $G^{\prime}$. After
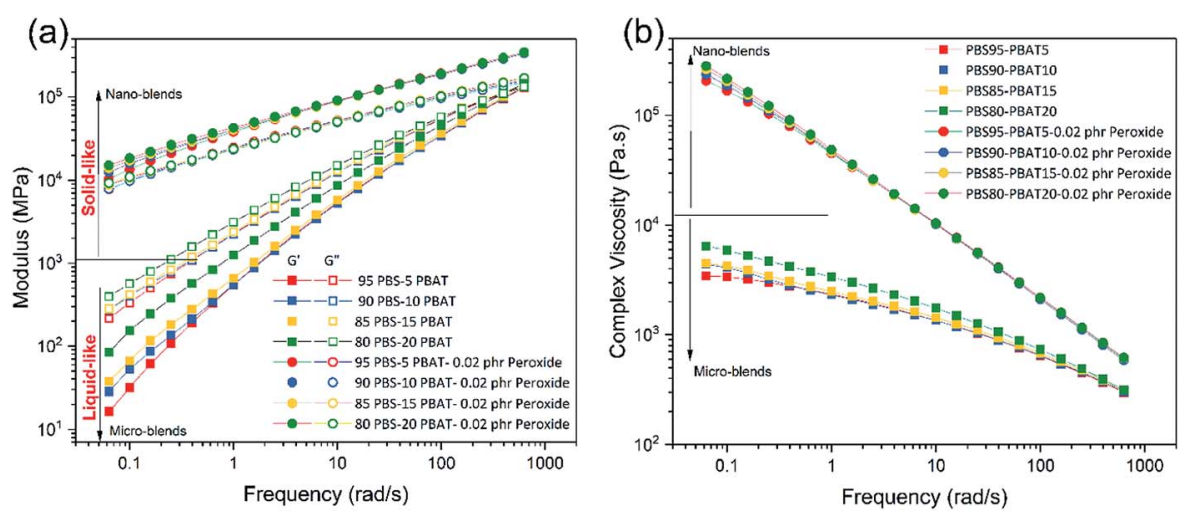

Fig. 3 High melt strength of the nano-blends revealed by rheological studies at $190{ }^{\circ} \mathrm{C}$. (a) The dependence of storage modulus $\left(\mathrm{G}^{\prime}\right)$ and loss modulus $\left(G^{\prime \prime}\right)$ on frequency; (b) the dependence of complex viscosity on frequency. 


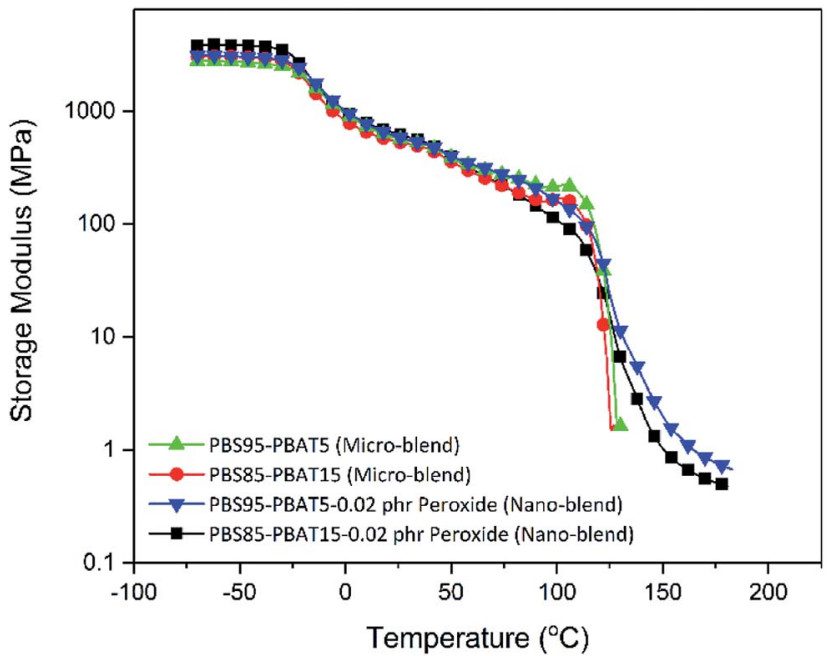

Fig. 4 Improved high temperature creep ability of nano-blends revealed by dynamic mechanical analysis - dual-cantilever frequency sweep.

peroxide curing, both the $\mathrm{G}^{\prime}$ and $\mathrm{G}^{\prime \prime}$ increase and are much higher than the micro-blends. More importantly is that the $\mathrm{G}^{\prime}$ is higher that the $\mathrm{G}^{\prime \prime}$ in the nano-blends (solid-like behaviour), indicating the improved elasticity of the melt. The solid-like viscoelastic reflects that the nano-blends possess high melt strength. ${ }^{29}$ The complex viscosity is also improved dramatically with the introduction of peroxide, as shown in Fig. 3b. Also, because of the branching and improved compatibility, the blends show strong shear shinning behaviour in the whole frequency range, indicating the disentanglement of the molecular chains in the testing frequency, especially in the long time scale (low frequency). ${ }^{30}$ It's suggested that the relaxation in long time scale is ascribed to the high entanglement branching chains formed during the reactive extrusion. ${ }^{31}$ The high melt strength ensures the broad processing window and an array of applications for the nano-blends, such as blowing,
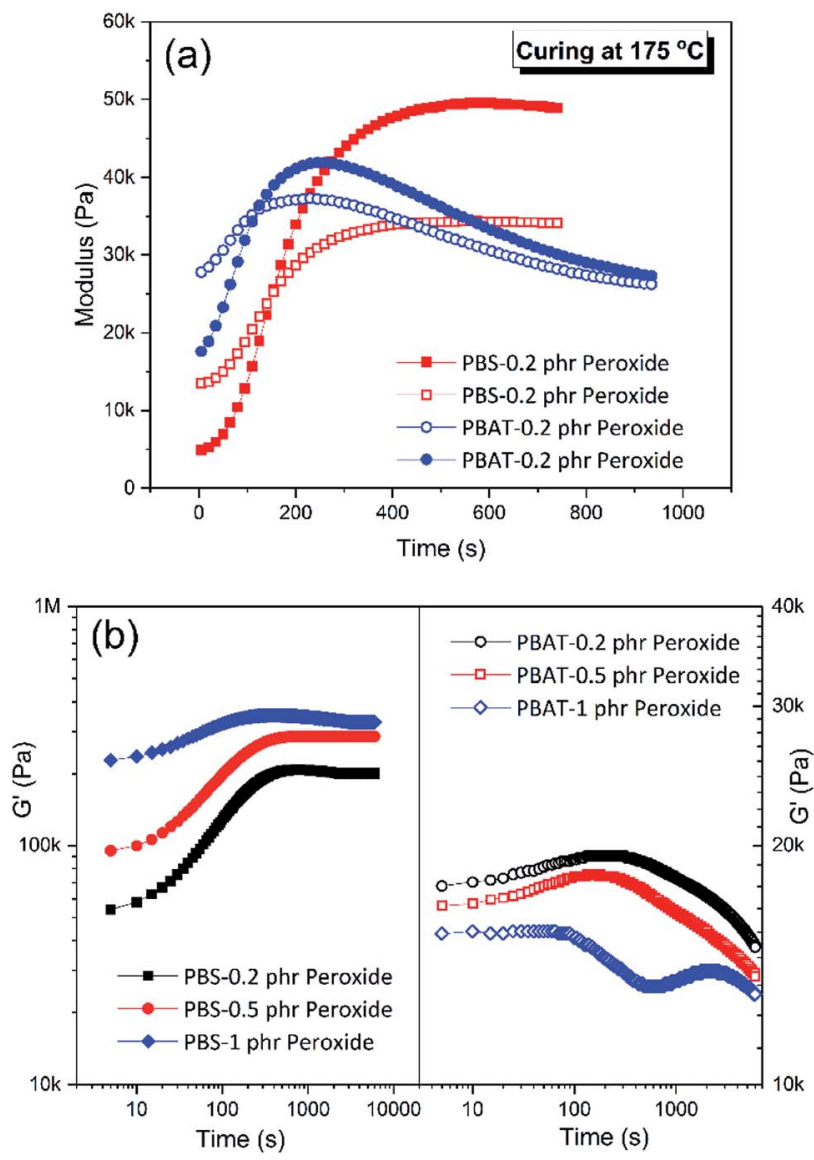

Fig. 6 Nano-structure PBS/PBAT binary blend formation mechanism. (a) curing of PBS and PBAT with $0.2 \mathrm{phr}$ peroxide at $175^{\circ} \mathrm{C}$ in the rheometer (solid: storage modulus; hollow: loss modulus); (b) the evolution of storage modulus (at $6.28 \mathrm{rad} \mathrm{s}^{-1}$ ) on time in the continue frequency sweep for PBS and PBAT/peroxide at $175^{\circ} \mathrm{C}$.

thermoforming, and foaming to replace the widely used nonbiodegradable petro-based plastics, expanding their applications in packaging market. Resistance to high temperature
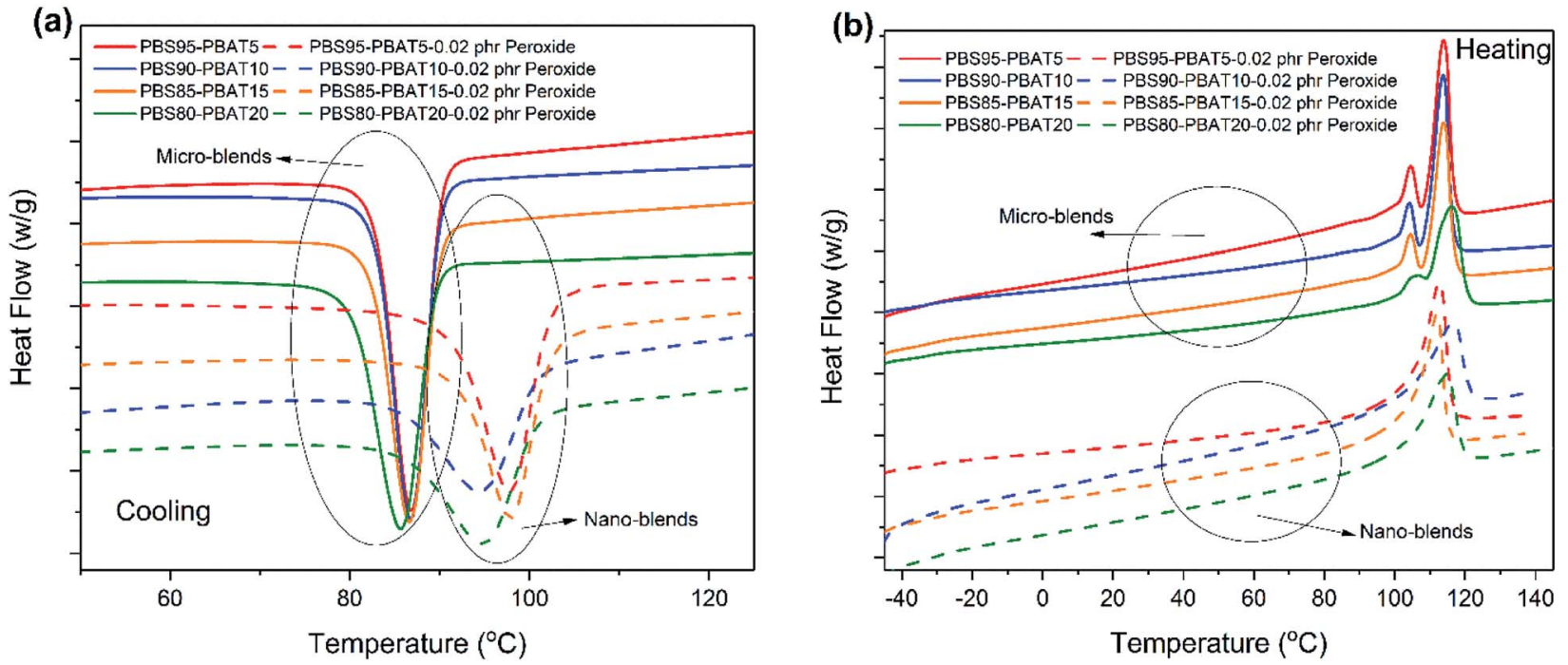

Fig. 5 Crystallization and melting behavior of the nano-PBS/PBAT blends revealed by DSC curves: (a) cooling at $10^{\circ} \mathrm{C}$ min ${ }^{-1}$; (b) heating at $10{ }^{\circ} \mathrm{C} \min ^{-1}$. 

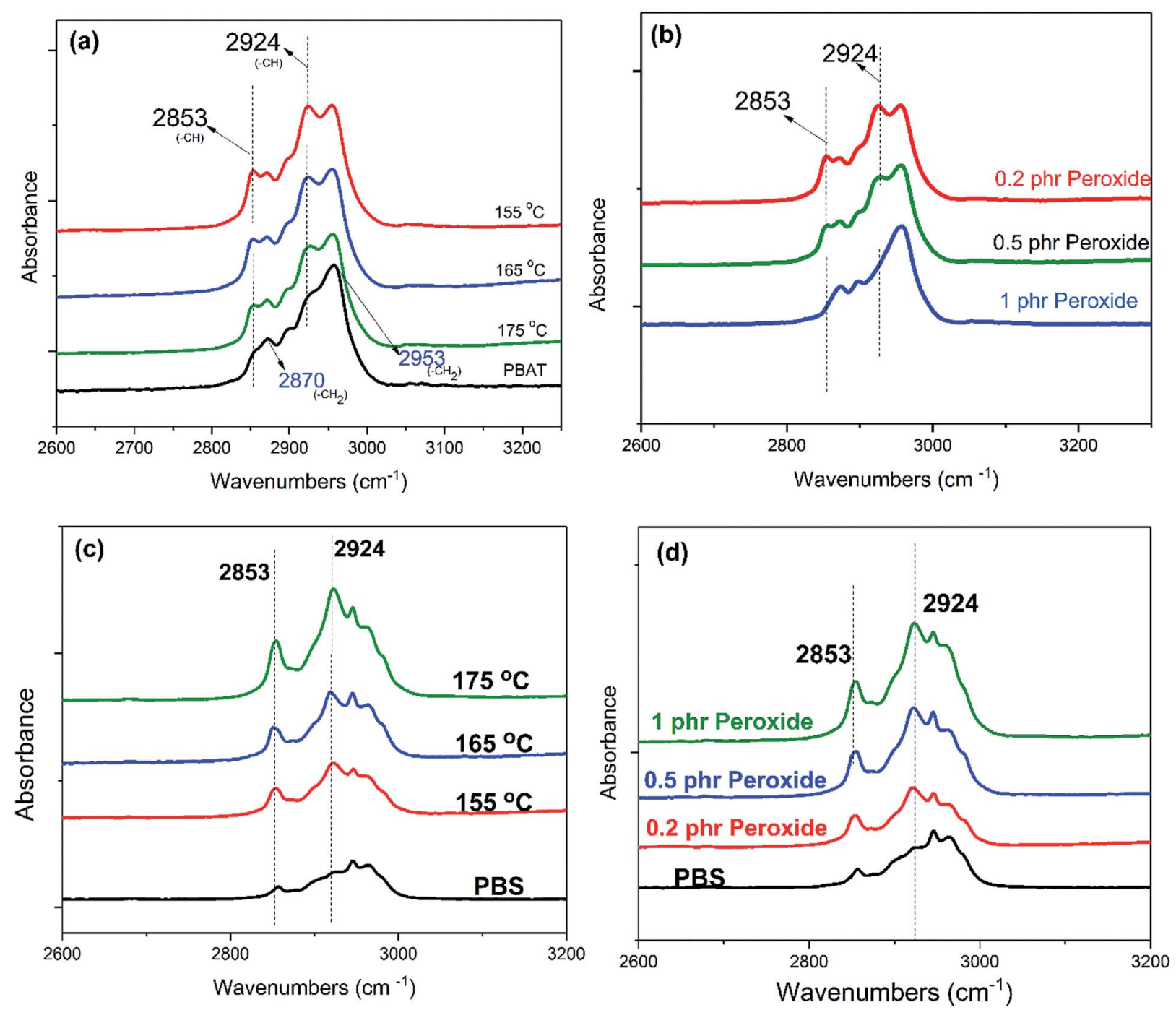

Fig. 7 The influence of peroxide concentrations and temperature on the chain structures of PBS and PBAT: (a) PBAT/0.2 phr peroxide at different temperatures; (b) PBAT/peroxide at $175^{\circ} \mathrm{C}$ with different peroxide contents; (c) PBS/0.2 phr peroxide at different temperatures; (d) PBS/peroxide at $175^{\circ} \mathrm{C}$ with different peroxide contents.

creep properties is also an important parameter for material to evaluate its dimensional stability at high temperature. The nano-blends possess high resistance to high temperature creep compared to the traditional micro-blends from the DMA testing shown in Fig. 4. It can be found that the storage modulus of the nano-blends declines slowly at temperature higher than the melting point of PBS $\left(114^{\circ} \mathrm{C}\right)$. In contrast, the storage modulus of the micro-blends without reaction decrease to zero directly at the melting point of PBS. The high temperature resistant of the nano-blends is believed to result from the high levers of entanglement of the molecular chains as revealed by the rheological studies.

The crystallization behaviour of the micro and nano-blends is studied by DSC and shown in Fig. 5. Compared to that of the micro-blends, the crystallization temperature has been increased after dispersing PBAT into nano-scale, as shown in Fig. 5a. It indicates that the nano-dispersed PBAT can be act as a more efficient nucleation agent for PBS, the same phenomenon has been observed in the previous research on nano- blends of poly(vinylidene fluoride) (PVDF)/acrylic rubber (ACM). ${ }^{32}$ It can also be found that the double-melting peaks in the micro-blends are disappeared and transformed into one high temperature melting peak in nano-blends, as shown in Fig. 5b, which resulted from the improved perfection of the crystals in nano-blends, such as smaller crystal size or fully growth crystals. ${ }^{33}$ It is suggested that the more perfect crystals also contribute the higher modulus in nano-blends. The crystallinity of the blends is shown in Fig. S2. $\dagger$ The crystallinity of the nano-blends reaches highest at $15 \mathrm{wt} \%$ PBAT is probably resulted from the large formation of the nano-lamina structure in the blends, as shown by the TEM in Fig. 1c.

\section{Nano-blends formation mechanism}

The aim of this work is to obtain PBS-PBAT co-polymer to improve the compatibility of different phases by a reactive extrusion process. Unfortunately, this reaction is difficult to control; it is difficult to obtain enough co-polymer but not over- 
crosslinked or decomposed homopolymers in which the large formation of block co-polymers is the guaranteeing to decrease the size of dispersed phase into nano-scale. To solve this problem, we start from two biodegradable polymers which possess similar molecular structures and close radical reaction rates: PBS, a homo-polymer consisting of butylene succinate, and PBAT which can be regarded as a co-polymer of butylene succinate and dimethyl terephthalate. In other words, the polymers chosen here are a homo-polymer A and a co-polymer with structure A-B, similar to the former discussed HIPS. ${ }^{4}$ Although the PBS and PBAT have similar chain segments and close glass transition temperature $\left(T_{\mathrm{g}}\right)$ (shown as DMA testing in Fig. S3†), our research on morphology, completed by SEM (Fig. S4 $\dagger$ ), shows that the blends are partially compatible which form co-continuous structure with macroscopically noticeable phase separation at $15 \mathrm{wt} \%$ PBAT.

The reactivity of PBS/PBAT resulted from free radical attack on the hydrogen atoms along the hydrocarbon skeleton. The free radicals which are generated by peroxide decomposition attacked the labile protons on the secondary carbon sites of PBS/PBAT. The free radicals of PBS/PBAT underwent either grafting or chain cleavage degradation. ${ }^{34}$ Furthermore, due to the high instability and reactivity of secondary free radicals, the grafting and degradation processes are extremely fast at high temperatures (>melting temperature) and led to either over cross-linking or serious degradation, respectively. While the over cross-linking of one phase in the reactive extrusion led to the phase separation as micelles, ${ }^{35}$ consequently nanostructures could not be obtained. Thus, stabilization and consumption of free radicals in favor of branching on the backbone of the chains is most important to obtain stable nanostructures rather than experiencing over cross-linking or serious chain degradation. ${ }^{21}$ Suppressing over cross-linking through decreasing the amount of initiators is the key factor in this method. However, to obtain enough reacted co-polymers in the final blends, which increased the interfacial entropy to improve the miscibility of the components, a proper amount of initiator should be used. Our hypothesis is that this complication is overcome by the complementary reaction between the PBS, PBAT and the low dose radical initiator. This ensured the formation of large amounts of PBS-PBAT co-polymer rather than over-crosslinked PBS or degraded PBAT. Other than suppressing over-crosslink or thermal decomposition, another difficulty of free radical grafting arose from the competition between co-polymer formation and homopolymers crosslinking. It is suggested that this obstacle could be overcome by a close reaction rate between the PBS/PBAT and free radicals. To verify this, we simulated the reaction between PBS, PBAT and the radical initiator with different amounts at various temperatures in a rheometer. The rheometer is used to detect the chain structure evolution by real-time monitoring of the modulus variation.

Firstly, the dependence of modulus on reaction time for PBS/ peroxide and PBAT/peroxide demonstrated in Fig. 6a reveals the possible reaction between the polymers and peroxide. The evolution of the modulus with time shows that the two polymers possess a fast and close reaction rate. It is known that the reaction rates measured in homogeneous melts are close to that of rates under mixing. ${ }^{36}$ The sharp increase of $\mathrm{G}^{\prime}$ at $175{ }^{\circ} \mathrm{C}$ (Fig. 6a) during crosslinking of homo-polymers in the rheometer shows that the reaction took place quickly, even under static conditions. Also, the kinetics of reaction are very similar for both materials as indicated by the identical $\mathrm{G}^{\prime}$ initial slopes ${ }^{37}$ (Fig. 6a). The fast and close coupling reaction plays a key role during the PBS/PBAT co-polymer formation rather than crosslinked homopolymers, while the amounts of co-polymer work as in situ compatibilizer to promote stability of the nanostructures.

Besides the partial compatibility and fast/close reaction rate of the two polymers with peroxide, more importantly, the dependence of peroxide concentrations on the reaction of these two polymers are totally different. When the peroxide concentrations are increased it is found that the equilibrium storage modulus of PBAT decreased with increasing peroxide concentrations as shown in Fig. 6b. This resulted from the more serious degradation of the PBAT chains caused by more free radicals, rather than increased crosslinking. The extent of crosslinking in PBS increases with increasing peroxide concentrations as expected; which is reflected from the increased equilibrium storage modulus in the continuous time sweeps shown in Fig. 6b. The above description of the dependence of crosslink or chain degradation on peroxide contents and temperature is also verified by the FTIR studies.

The evolution of the structure of PBAT with the temperature and peroxide concentrations is revealed by FTIR and shown in Fig. 7a and b. Among the structure-related peaks, two peaks at 2853 and $2924 \mathrm{~cm}^{-1}$ appear in the range of $2800-3000 \mathrm{~cm}^{-1}$ are used to follow the structure evolution of the polymer chains

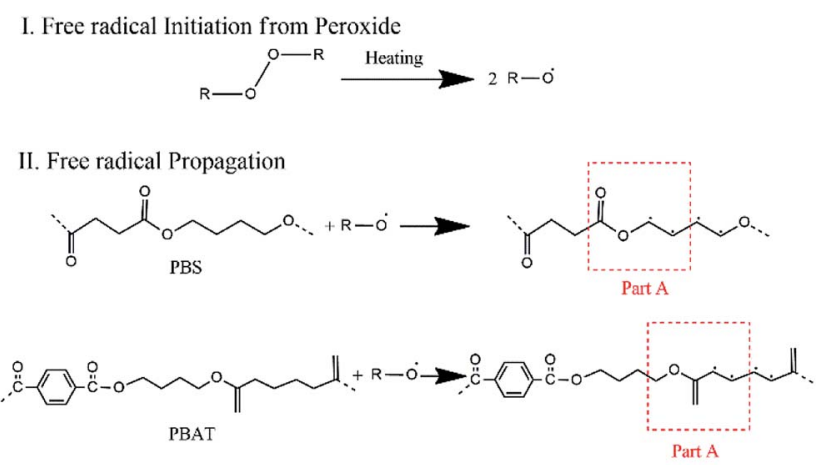

III. Free radical Termination

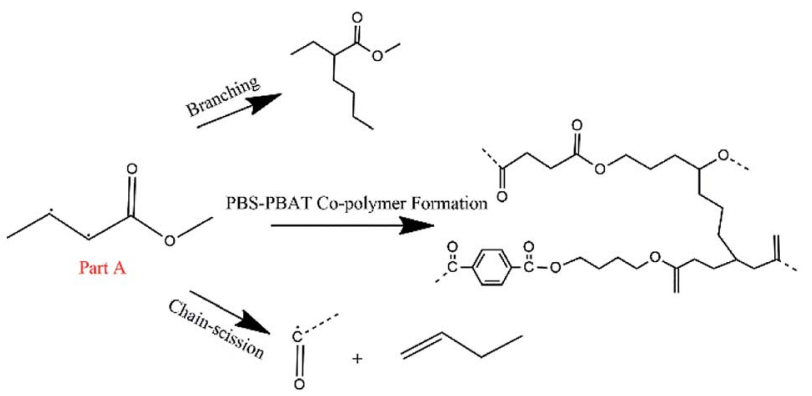

Fig. 8 The reaction mechanism between the biodegradable polymers and free radical initiators. 
during the reaction with free radicals. These two peaks, which are caused by the vibration of $-\mathrm{CH}$ group,$^{38}$ are believed to be from the free radicals attract of methylene on the carbon backbone of PBS and PBAT chains. Since the $-\mathrm{CH}_{2}$ on the backbone of PBS and PBAT will be attacked by the free radicals and transformed into $-\mathrm{CH}$. The peak intensity can be used to demonstrate the degree of the crosslinking or branching. The new peak intensity of PBAT/peroxide decreases with increasing peroxide concentrations and temperature, as shown in Fig. 7a and $\mathrm{b}$. It means that with increasing peroxide concentrations/ temperature, less $-\mathrm{CH}_{2}$ is attracted by the free radicals, leading to the limited branching of the molecular chains at high peroxide concentrations. The evolution of the structure of PBS with the temperature and peroxide concentrations is also revealed by FTIR and shown in Fig. $7 \mathrm{c}$ and $\mathrm{d}$. As indicated by the FTIR spectrums, the increasing intensity of the PBS/peroxide peaks with increasing peroxide content and temperature means that more $-\mathrm{CH}_{2}$ groups are attracted by the free radicals and transformed to $-\mathrm{CH}$, and finally more branching/ crosslinking points are generated on the main chains, leading to the high crosslinking or branching. This is the first time we observed from FTIR spectrum showing the influence of peroxide concentration on the structure evolutions of polymers in free radical reactions. The reaction mechanism based on the FTIR spectrum evolution is illustrated in Fig. 8.

Through decreasing the concentration of peroxide into small amount, both the over-crosslink of PBS and serious chain scission of PBAT are suppressed. Our hypothesis is that these complementary reactions between the PBS, PBAT and the low dose radical initiator ensured the formation of large amounts of PBS-PBAT co-polymer rather than over-crosslinked PBS or thermal degraded PBAT, which is the key factor to form nanoblends in this method. The similar chemical structure, fast and close reaction rate, as well as the complementary reaction worked together in the formation of nano-structures in the blends via reactive extrusion.

To verify the peroxide concentrations on the formation morphology of these two blends, the morphology of the PBS/ PBAT blends with higher peroxide ( $0.2 \mathrm{phr}$ used here) and lower peroxide $(0.007 \mathrm{phr})$ is observed and compared to the nano-blends (0.02 phr peroxide), as shown in Fig. 9. By introducing different amounts of peroxide into PBS95/PBAT5 blends, it is found that only nano-structure could be formed with the appropriate peroxide contents (0.02 phr here). At $0.2 \mathrm{phr}$
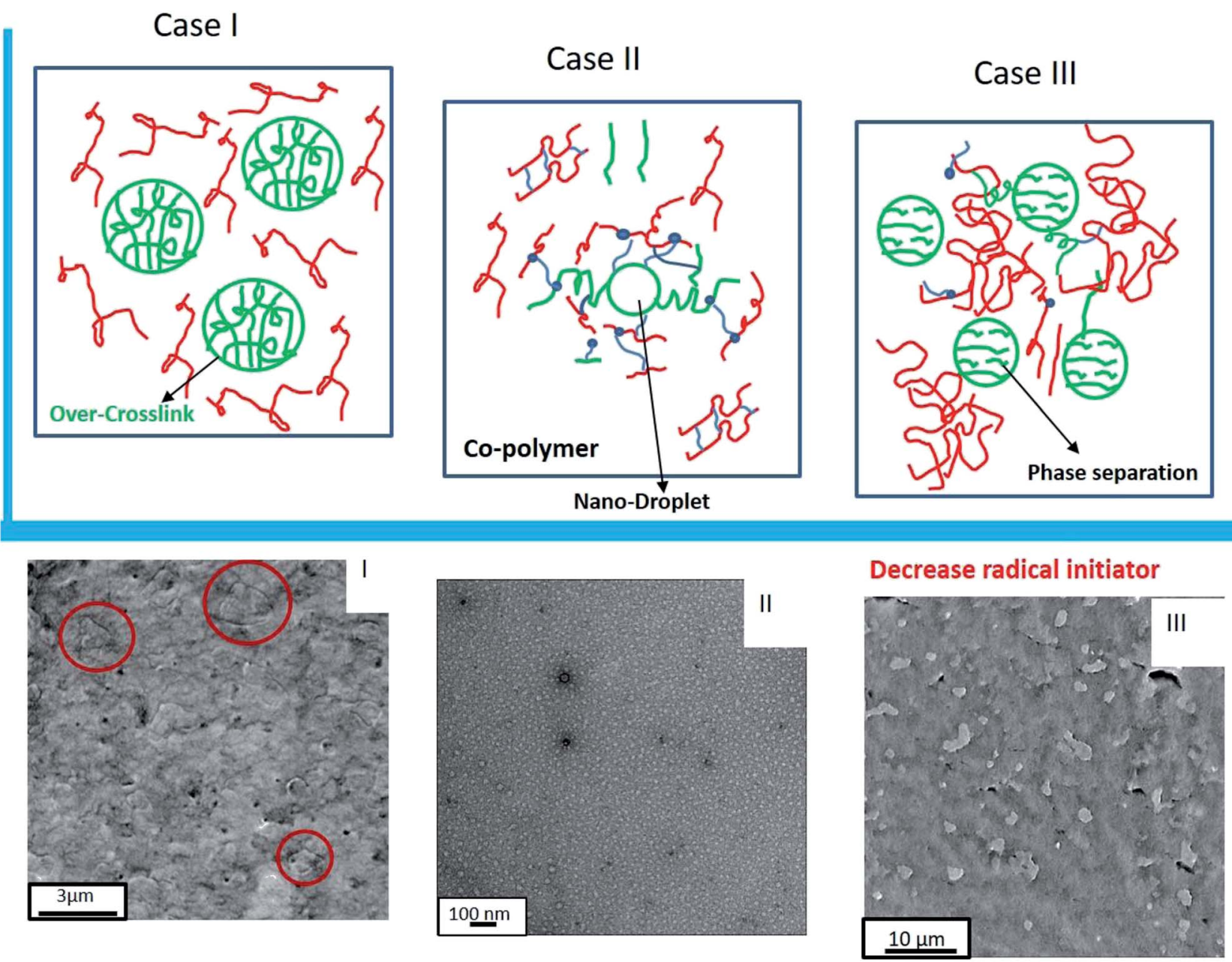

Fig. 9 Schematic diagram and morphology observation of the structure evolution of the PBS95/PBAT5/peroxide blends on peroxide concentrations: (I) $C_{\text {peroxide }}=0.2 \mathrm{phr}$; (II) $C_{\text {peroxide }}=0.02 \mathrm{phr}$; (III) $C_{\text {peroxide }}=0.007 \mathrm{phr}$. 
peroxide, the blend is over-crosslinked and difficult to meltprocess (the gel contents reach as high as $38 \%$ ), leading to the phase separation in micro-scale, as indicated by the red circle in the SEM photo (high peroxide concentrations) in Fig. 9I. It's suggested that the micro-separated droplet is the over-crosslinked micelles, ${ }^{35}$ as indicated by case I; at low peroxide ( $0.007 \mathrm{phr})$, the PBS-PBAT co-polymers are deficient because of the less of free radicals. Therefore, the compatibility cannot be improved with small amount of peroxide, leading to the micro-dispersed PBAT in the extruded materials, as indicated by case III in Fig. 9. From the SEM in Fig. 9III, the PBAT dispersed in the PBS as a droplet with diameter of $\sim 2 \mu \mathrm{m}$, reflecting the phase separation.

As discussed, the formation of a large amount of co-polymer is the guarantee of the nano-dispersed of PBAT in PBS. To observe the morphology, AFM is applied to check the nanoblend structures. The AFM photographs of PBS95/PBAT5/0.02 $\mathrm{phr}$ peroxide and PBS85/PBAT15/0.02 phr peroxide which show two different structures in TEM are given in Fig. 10. At low PBAT contents (shown as Fig. 10a and c), finely dispersed phase (low modulus PBAT indicated as dark points) as small as $80 \mathrm{~nm}$ can be clearly seen. Since the large amounts of the co-polymer are formed and tightly surrounded the PBAT phase, the size of the dispersed phase seems like larger than that shown in TEM. The brown phase detected in the AFM technology surrounding the low modulus PBAT is believed to be the copolymer phase which possesses tapping modulus between neat PBS and PBAT. Thus, a core-shell structure with high affinity to the matrix are successfully formed in the nanoblends, which is believed to attribute the super-toughness of PBS. With increasing PBAT to $15 \mathrm{wt} \%$, it can be found the dispersed morphology has been greatly changed. The AFM phase contrast micrograph of this blend is shown in Fig. 10b. No obvious dispersed PBAT phase can be found in this blends because of the large formation of co-polymers, same as the TEM observations. From the $\log$ (modulus) diagram shown in

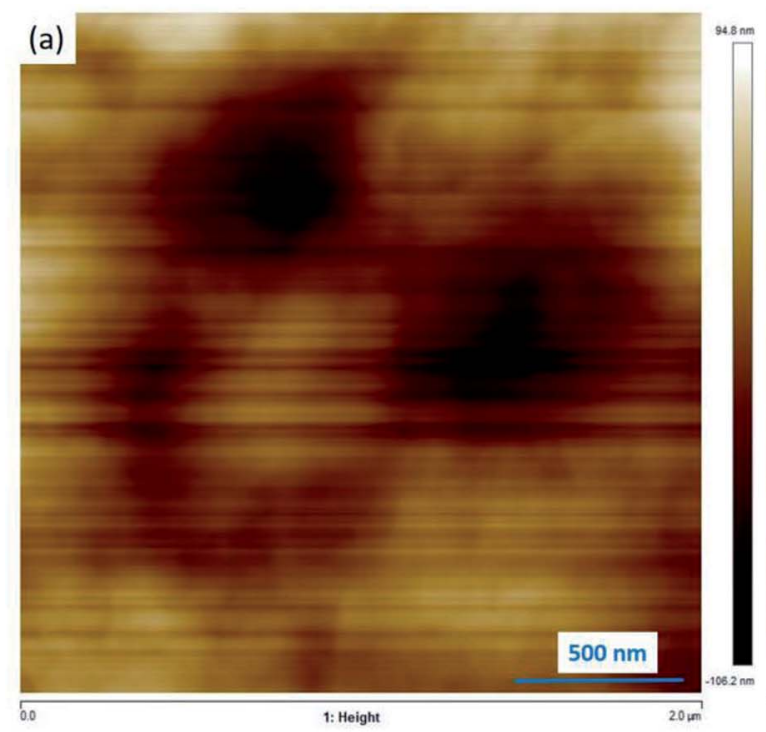

(b)
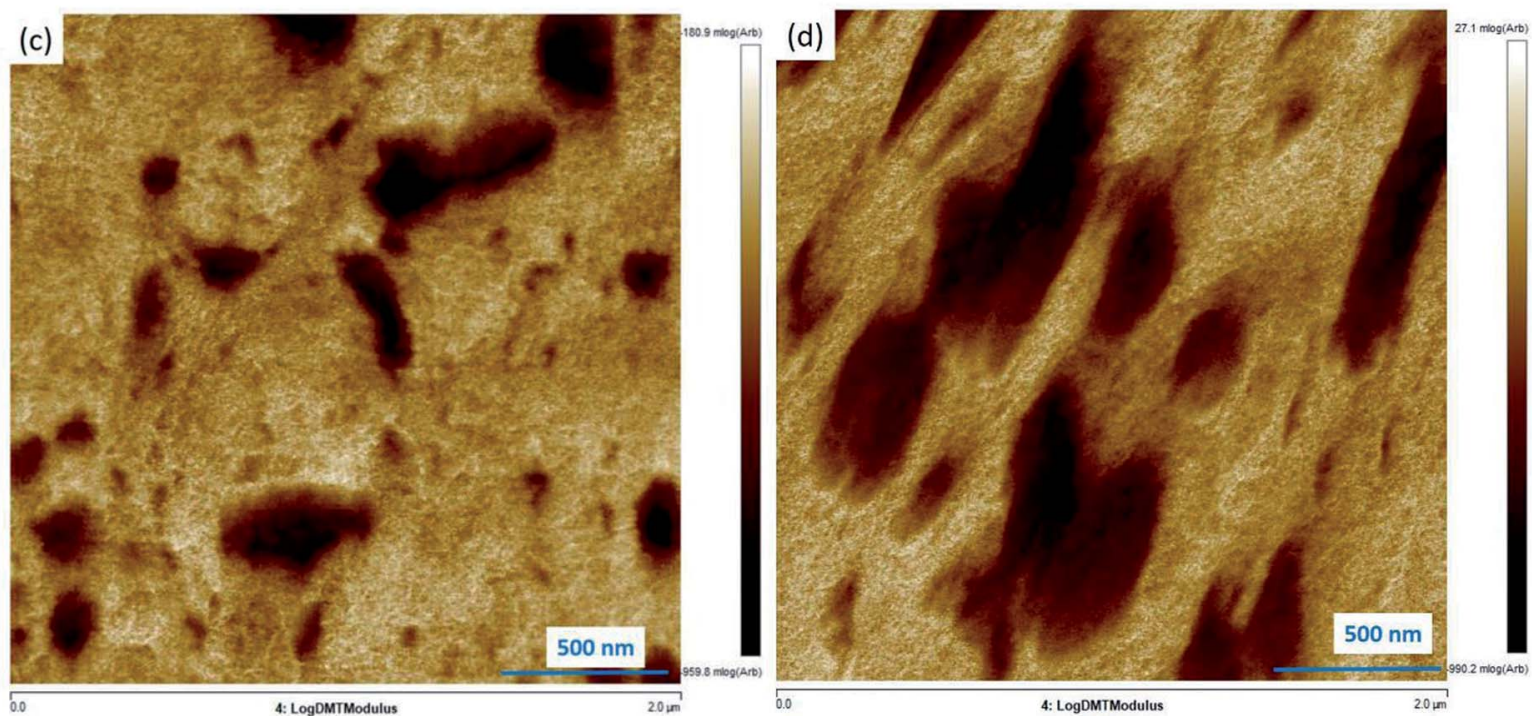

Fig. 10 The AFM photograph of the PBS/PBAT nano-blends: height morphology: (a) PBS95/PBAT5/0.02 phr peroxide, (b) PBS85/PBAT15/0.02 phr peroxide; log modulus morphology: (c) PBS95/PBAT5/0.02 phr peroxide, (d) PBS85/PBAT15/0.02 phr peroxide. 
Fig. 10d, elongated strips with core-shell structures are observed in the PBS matrix. The low modulus PBAT (shown as dark in AFM) parts are surrounded by large amounts of copolymer phase shown as brown parts in the diagram. It is suggested that the co-polymers exhibit as nano-lamina structures in the TEM photos as indicated by Fig. 1c.

\section{Conclusion}

In summary, our research found that nano-scale blends with high performance which are unattainable from classical microblends can be obtained through reactive extrusion by selecting appropriate polymer pairs. Super-toughened PBS/PBAT nanoblends are prepared with small amount of PBAT dispersed as nano-scale phase in the continuous PBS phase. The nanoblends show higher modulus compared to the neat PBS, without sacrifice of the stiffness when introducing low modulus toughening PBAT. A 12-fold improvement in notched impact strength and 1.2-fold increasing in modulus has been achieved in the nano-blends. The DSC studies shown that, the PBAT can be acted as a nucleating agent for PBS to increase the crystallization temperature and crystal perfection when dispersing the toughening phase into nano-scale. The morphology observation by AFM show the formation of large amounts of co-polymers surrounding the toughening phase, leading to the fine dispersion of the PBAT.

In reactive extrusion, favoring the formation of co-polymers rather than self-crosslinking or chain decomposition is the key factor in improving the performance of polymer blends, including thermomechanical performance and processability. The in situ rheology time sweeping monitoring and FTIR in this study indicates the important considerations concerning the reaction rates and the influence of peroxide amounts on the reaction mechanism of polymers in reactive extrusions, to copolymer formations. The final dispersion morphology of the polymer blends is influenced by the peroxide amounts in the reactive extrusion.

The cost-effective preparation method used in this work does not require any solvents or extra catalysts and the extremely low amount of peroxide initiator used ensures the green processing of the products. The nano-structured materials engineered from binary biodegradable polymer blends show super toughness with high modulus and improved melt strength. The newly developed materials show promising application in sustainable packaging use where high toughness, high melt strength and potential biodegradability are required. Since the materials are developed from two biodegradable polymers and very low concentration of peroxide is used here, it is expected to be biodegradable. However, the detailed composability studies need to be finished to claim the biodegradability of these developed nano-blends.

\section{Conflicts of interest}

There are no conflicts to declare.

\section{Acknowledgements}

The authors would like to thank the following for their financial support to carry out this research: (1) the Ontario Ministry of Agriculture, Food and Rural Affairs (OMAFRA)/University of Guelph - Bioeconomy for Industrial Uses Research Program (Project \# 030054) and the OMAFRA/University of Guelph Product Development and Enhancement through Value Chains Theme (Project \# 030146); (2) the Agriculture and Agri-Food Canada (AAFC) and Competitive Green Technologies through AgriInnovation Program project (Project \# 052882 and 051910); and (3) the Natural Sciences and Engineering Research Council (NSERC), Canada Discovery Grants (Project \# 401111 and 400320).

\section{Notes and references}

1 C. Arends, Polymer toughening, CRC Press, 1996, vol. 30.

2 M. Szwarc, 'Living' Polymers, Nature, 1956, 178, 1168.

3 J. L. Amos, The SPE international award address-1973 the development of impact polystyrene-a review, Polym. Eng. Sci., 1974, 14(1), 1-11.

4 D. J. Kinning, K. I. Winey and E. L. Thomas, Structural transitions from spherical to nonspherical micelles in blends of poly (styrene-butadiene) diblock copolymer and polystyrene homopolymers, Macromolecules, 1988, 21(12), 3502-3506.

5 A. Jimenez, M. Peltzer, and R. Ruseckaite, Poly (lactic acid) science and technology: processing, properties, additives and applications, Royal Society of Chemistry, London, 2015.

6 M. Meier, Sustainable polymers: reduced environmental impact, renewable raw materials and catalysis, Green Chem., 2014, 16(4), 1672.

$7 \mathrm{M}$. Rabnawaz, et al., A roadmap towards green packaging: the current status and future outlook for polyesters in the packaging industry, Green Chem., 2017, 19(20), 4737-4753.

8 R. A. Gross and B. Kalra, Biodegradable Polymers for the Environment, Science, 2002, 297, 4.

9 A. K. Mohanty, M. Misra and G. Hinrichsen, Biofibres, biodegradable polymers and biocomposites: An overview, Macromol. Mater. Eng., 2000, 276-277(1), 1-24.

10 S. Sahoo, M. Misra and A. K. Mohanty, Enhanced properties of lignin-based biodegradable polymer composites using injection moulding process, Composites, Part A, 2011, 42(11), 1710-1718.

11 L. Leibler, Nanostructured plastics: Joys of self-assembling, Prog. Polym. Sci., 2005, 30(8), 898-914.

12 U. Sundararaj and C. Macosko, Drop breakup and coalescence in polymer blends: the effects of concentration and compatibilization, Macromolecules, 1995, 28(8), 26472657.

13 Y. L. Hiroshi Shimizu, A. Kaito and H. Sano, Formation of Nanostructured PVDF/PA11 Blends Using High-Shear Processing, Macromolecules, 2005, 38, 7880-7883.

$14 \mathrm{Y}$. Li and H. Shimizu, Fabrication of nanostructured polycarbonate/poly(methyl methacrylate) blends with 
improved optical and mechanical properties by high-shear processing, Polym. Eng. Sci., 2011, 51(7), 1437-1445.

15 A.-V. Ruzette and L. Leibler, Block copolymers in tomorrow's plastics, Nat. Mater., 2005, 4(1), 19-31.

16 G.-H. Hu, H. Cartier and C. Plummer, Reactive Extrusion: Toward Nanoblends, Macromolecules, 1999, 32(14), 47134718.

17 Y. Ji, et al., A Novel Approach to the Preparation of Nanoblends of Poly(2,6-dimethyl-1,4-phenylene oxide)/ Polyamide 6, Macromol. Rapid Commun., 2005, 26(2), 116120.

18 R. Bhardwaj and A. K. Mohanty, Modification of Brittle Polylactide by Novel Hyperbranched polymer based nanostructure, Biomacromolecules, 2007, 8, 2476-2484.

19 P. A. Bhadane, et al., Morphology Development and Interfacial Erosion in Reactive Polymer Blending, Macromolecules, 2008, 41(20), 7549-7559.

20 C. Tzoganakis, Reactive extrusion of polymers: A review, Adv. Polym. Technol., 1989, 9(4), 321-330.

21 H. Pernot, M. Baumert and L. Leibler, Design and properties of co-continuous nanostructured polymers by reactive blending, Nat. Mater., 2002, 1(1), 54.

22 R. Muthuraj, M. Misra and A. K. Mohanty, Biodegradable Poly(butylene succinate) and Poly(butylene adipate-coterephthalate) Blends: Reactive Extrusion and Performance Evaluation, J. Polym. Environ., 2014, 22(3), 336-349.

23 R. Muthuraj, M. Misra and A. K. Mohanty, Hydrolytic degradation of biodegradable polyesters under simulated environmental conditions, J. Appl. Polym. Sci., 2015, 132(27), 42189.

24 F. Wu, M. Misra and A. K. Mohanty, Super Toughened Poly (Lactic Acid) (PLA)-based Reactive Ternary Blends via Enhancing Interfacial Compatibility, ACS Omega, 2018, DOI: $10.1021 /$ acsomega.8b02587.

25 P. J. Carreau, Rheological equations from molecular network theories, Trans. Soc. Rheol., 1972, 16(1), 99-127.
26 Z. Gan, et al., Solid-State Microstructures, Thermal Properties, and Crystallization of Biodegradable Poly(butylene succinate) (PBS) and Its Copolyesters, Biomacromolecules, 2001, 2(2), 605-613.

27 Z. Tadmor and C. G. Gogos, Principles of Polymer Processing, A John Wiley \& Sons, Inc., United States of America, 2006, p. 982.

$28 \mathrm{~S}$. Wu, Chain structure, phase morphology, and toughness relationships in polymers and blends, Polym. Eng. Sci., 1990, 30(13), 753-761.

29 A. I. Leonov, On the rheology of filled polymers, J. Rheol., 1990, 34(7), 1039-1068.

30 J. D. Ferry and J. D. Ferry, Viscoelastic properties of polymers, John Wiley \& Sons, 1980.

$31 \mathrm{~J}$. Liu, et al., Long chain branching polylactide: Structures and properties, Polymer, 2010, 51(22), 5186-5197.

32 Y. Li, et al., Nanostructured Poly(vinylidene fluoride) Materials by Melt Blending with Several Percent of Acrylic Rubber, Macromolecules, 2008, 41(9), 3120-3124.

33 Z. Kulinski and E. Piorkowska, Crystallization, structure and properties of plasticized poly(l-lactide), Polymer, 2005, 46(23), 10290-10300.

34 J.-M. Raquez, R. Narayan and P. Dubois, Recent Advances in Reactive Extrusion Processing of Biodegradable PolymerBased Compositions, Macromol. Mater. Eng., 2008, 293(6), 447-470.

35 A. Adedeji, S. Lyu and C. W. Macosko, Block Copolymers in Homopolymer Blends: Interface vs. Micelles, Macromolecules, 2001, 34(25), 8663-8668.

36 C. W. Macosko, H. K. Jeon and T. R. Hoye, Reactions at polymer-polymer interfaces for blend compatibilization, Prog. Polym. Sci., 2005, 30(8), 939-947.

37 L. Imbernon, et al., Chemically crosslinked yet reprocessable epoxidized natural rubber via thermo-activated disulfide rearrangements, Polym. Chem., 2015, 6(23), 4271-4278.

38 I. Fleming and D. H. Williams, Spectroscopic methods in organic chemistry, 1966. 